\title{
CoVID-19 Boundary work during COVID-19: The transformation of research review and set-up
}

\author{
Authors: Cecilia Vindrola-Padros, ${ }^{A}$ Daniel Herron ${ }^{B}$ and Nick McNally ${ }^{C}$
}

\section{Background and aims}

The rapid setting up of research during the COVID-19 pandemic led to changes in ways of working within research organisations. The aim of this study was to examine the experiences of staff involved in the research review and set-up system at a large NHS and university partnership in the UK through the lens of boundary theory.

\section{Methods}

We carried out a rapid qualitative appraisal based on telephone interviews $(n=25)$ to explore how staff experienced the research review and set-up system during the pandemic.

\section{Results}

In light of the pressures created by the pandemic, the boundaries established to set up distinct groups and responsibilities were modified to allow for different ways of working. Some of the new structures and processes were seen positively and brought groups that previously worked at a distance closer together.

\section{Conclusions}

The reconceptualisation of relations within the research system during the pandemic added more fluidity to ways of working within the research office and contributed to closer working interactions and an expanded team ethos.

KEYWORDS: COVID-19, research office, boundaries

DOI: $10.7861 /$ fhj.2020-0262

\section{Introduction}

The COVID-19 pandemic has put unprecedented pressure on research systems and research offices around the world. The need to rapidly review and set up research that could make a contribution to the management of the pandemic has meant that many research offices have had to develop clear and efficient processes for fast-tracking COVID-19 applications, while making difficult decisions about studies on other conditions. These new

Authors: Asenior research fellow, University College London, London, UK; Bhead of research innovation, NIHR UCLH Biomedical Research Centre, London, UK; ' managing director, research UCLH/ UC, NIHR UCLH Biomedical Research Centre, London, UK processes have generated changes in ways of working within research organisations, affecting structures, decision-making processes and relations. In this paper, we describe the changes that were required to facilitate the rapid review and set-up of research during the first wave of the COVID-19 pandemic in the UK.

Previous research has already pointed to the dynamic and hybrid role of research offices ${ }^{1}$ and has highlighted the benefits of using the lens of boundary theory to understand their daily practices. ${ }^{2}$ Within this framework, 'boundary spanners' have roles that require them to involve multiple organisations or sectors within organisations or to cut across disciplines or professional groups, regularly negotiating pre-established boundaries in order to transfer skills, knowledge or information across them. ${ }^{3}$ Boundary spanning at individual and team level has been identified as a feature of good practice in research offices, enabling collaborative relations that facilitate study review and set-up. ${ }^{4,5}$ However, in-depth descriptions of the practices entailed in this 'boundary work' remain a gap in the literature. Furthermore, most of the research has focused on boundary spanning, leaving out other aspects of boundary work, ${ }^{6}$ such as boundary making and boundary strengthening.

In this paper, we examine the experiences of a large joint (university and NHS) research office in the UK through the lens of boundary spanning, making and strengthening. We carried out a rapid qualitative appraisal to explore the active role played by joint research office staff, researchers and other staff involved in the research process in relation to both boundary spanning and strengthening and the everyday practices of (re)making boundaries. We focus on the adaptation of working practices in the context of pandemics to understand how research-active organisations are able to transform their ways of working in light of these new pressures.

\section{Setting}

The research office where we carried out this study, at University College London Hospitals NHS Foundation Trust and UCL, normally manages a portfolio of over 1,000 clinical research studies, one of the largest clinical research portfolios in the UK. The portfolio includes clinical trials of drugs and other interventions at all phases of evidence generation, as well as diagnostic studies and observational studies. The portfolio reflects the clinical and scientific expertise of the University College London Hospitals NHS Foundation Trust and its university partner, spanning multiple disease areas, 600 principal investigators (PIs) at any one time and multiple sponsors and funding organisations. 


\section{Questions guiding the evaluation}

The study was guided by the following questions:

$>$ What were the main changes in internal processes and working dynamics developed by the joint research office to deal with the demands generated by the COVID-19 pandemic?

$>$ How were boundaries spanned or strengthened as a result of these changes?

\section{Design}

We used a qualitative rapid appraisal design. ${ }^{7.8}$ Rapid appraisals were developed to collect and analyse data in a targeted way within limited timeframes and 'diagnose' a situation. ${ }^{9}$ A rapid appraisal design often combines two or more methods of data collection and then uses triangulation from different sources as a form of data validation..$^{10}$ It is based on an iterative process of collection and analysis, where 'the researchers begin with information collected in advance, and then progressively learn from each other and from information provided by semi-structured interviews and direct observations."11

\section{Data collection, recruitment and sampling}

The study was based on telephone interviews with joint research office staff $(n=10)$, academic and clinical researchers $(n=11)$ and staff from support departments $(n=4)$. Semi-structured interviews were conducted via telephone to explore the team-based models used by the joint research office during the COVID-19 pandemic, barriers encountered in the management of research, areas of good practice and processes that should remain after the pandemic.

Staff members were approached by the researchers via email and they were provided with a copy of the information sheet, which included details about the purpose, design, expectations, risks and benefits of the study. Staff were given 48 hours to decide if they would like to take part in the study. Staff who decided to take part in the study were asked to sign and email the researcher a consent form. The researcher then proceeded to arrange a telephone interview at a time that was convenient for them. Thirty potential participants were approached to take part in the study and 25 participated in the interview. The study was classified as a service evaluation according to the Health Research Authority (HRA) decision tool, so it did not need to be reviewed by a Research Ethics Committee.

\section{Data analysis}

The data were analysed using framework analysis. ${ }^{12}$ The interviewer took notes during the interviews and these were entered into a rapid assessment procedures (RAP) sheet. We have described the use of RAP sheets. ${ }^{13}$ The RAP sheet was reviewed by the UCL team to develop a framework. The framework was initially informed by the literature on boundary spanning roles in the context of research management, but it also included additional topics emerging from the data.

\section{Findings}

Our key findings can be divided into three broad themes: changes in structures and processes, changes in relations, and the reinforcement of boundaries.

\section{Structural and processual changes}

To establish capacity in the hospital for the care of COVID-19 patients and for a new portfolio of COVID-19 research, the joint research office instigated significant changes in relation to its internal structures and processes for reviewing, coordinating and setting up new studies. One of the most important early changes it had to make was a new policy to pause all studies unless they fell into the category of serious or life-threatening disease. This entailed the rapid development of a policy for approval by the Trust within 48 hours with clear criteria for distinguishing between studies that needed to be paused and those that could continue and the clear communication of this information to PIs. A COVID-19 response group was set up to oversee rapid implementation of the policy. This multidisciplinary group involved staff from the joint research office, with representatives from service support departments and significant research groups as well as patients. Staff from the joint research office also needed to communicate with PIs who required further explanation of the rationale for the policy and why their studies needed to be temporarily paused. Following the first wave, the focus of the group shifted in May 2020 to developing the policy for restarting the non-COVID clinical research portfolio and overseeing that activity.

Another major change was the creation of a COVID-19 strategy group. This group focused on coordinating the strategy for COVID-19 research, bringing together PIs, joint research office staff and staff from support departments to discuss these studies and to provide a strategic steer. One of the study participants described these groups as:

the COVID-19 research response group... which was the group to oversee the policy of pausing studies plus the implementation of it, anticipating that principal investigators who wanted to appeal or provide information as to why they felt their studies should carry on, so we sent that off, and the second committee alongside it, is what we call the COVID-19 research strategy group, which was designed to bring together the PIs with aspirations and interests in getting COVID studies off the ground, through which we had expected for COVID studies to come, and get a bit of an airing so that we could endorse and support, identify issues with getting COVID studies set up. (Senior manager, joint research office).

\section{Relational changes}

When asked about the impact of the changes described above on daily working relations, interview participants indicated that these changes had allowed them to develop closer working relations with their colleagues in the joint research office, PIs and staff in support departments who were authorised to determine local capacity for undertaking a study. In effect, the boundaries between the different teams within the joint research office as well as between the joint research office and academic and clinical departments became more permeable. As most of the communication was happening via telephone and social media channels such as WhatsApp, PIs felt they had unprecedented access to joint research office staff and could call them regularly for updates. Similarly, joint research office staff felt that they were not constrained by having to send emails only during office hours and could communicate with all relevant actors at any 
reasonable time via video conferencing calls or SMS. This enabled them to have quick calls with PIs and support department staff to confirm details about the study without the need to send a formal email:

I think quick communication with other teams is very, very important and this COVID situation has allowed us to do that. So sometime on, you know, I can just pick up the phone and call across or, you know, send them a message over the phone, or whatever. And I was able to get that answer from them; that was quite useful (contracts staff, joint research office).

Collaboration was identified as the key driver in the quick review and approval of studies:

...the reason why the processes are so complicated I think is because they are designed to be bulletproof and then, ensure the quality system is maintained and of course, I totally support that. But we have seen now that, with the right mixture of collaboration between all the members that are required for a study, and the right amount of being, following due diligence, you can ensure the quality in the same way and then making it faster. (Portfolio officer, joint research office.)

This quick turnaround was also facilitated by studies being reviewed in parallel by different teams within the joint research office, such as the teams managing contracts and finance. Prior to the pandemic, review processes had some degree of overlap, but still with some staggering of input from different teams, each of which have quite different expertise. Enhancing the parallel processing of studies during the pandemic was seen to save time:

Now we were overlapping a bit, so we were overlapping more the contracts with the costings review and they were working between them. Not necessarily meetings but it's true we were firing emails at the same time with different, with different teams, which made [...] communicating like almost live. (Portfolio officer, joint research office.)

The response group and strategy group were also identified as structures that promoted the interaction of relevant actors in a different way, as the aim of these groups was to generate a more rapid and open discussion with PIs about their studies and why these would be important for both the university and hospital and ultimately for the UK's research response to the pandemic. The response group also had patients as members and this was highlighted as a positive change by some study participants, as it enabled patient engagement in decision-making processes where such views had been previously difficult to incorporate. One of the patient members described their experience:

I saw my role as really important. It was a bit daunting at the beginning as we are not normally used to being in these roles. We are not experts and wanted to do a good job reviewing the studies. We also did not want to hold anyone up. [...] I think it was a good experience and we worked hard to make sure our ideas as patients came out right and were helpful.

Patient representatives spanned boundaries within the joint research office, by becoming active members of multiple decisionmaking groups.

\section{Boundary reinforcement}

It is important to note that not all study participants felt that boundaries had become more permeable. We were able to identify some examples of boundary reinforcement as well as the creation of boundaries that were not perceived as existing prior to the COVID-19 pandemic. While several of the PIs who were interviewed described positive experiences with the new review and set-up processes, some PIs still perceived the joint research office to be acting in its gatekeeping role, with processes that, in their opinion, delayed the study review and approval processes. Another important point made by some of the study participants related to decisions regarding membership of the specific COVID-19 research groups described above. The creation of new groups of staff and new working dynamics during the pandemic created a sense of new boundaries for some participants.

\section{Discussion}

The concept of boundaries has been frequently used in the social science literature to define the process of creating a distinction between different categories of places, people, time or situations. ${ }^{14}$ Boundaries are often seen as rigid and impermeable, with the need for objects or people to span them. Some authors have argued that, instead of considering boundaries as the object of analysis, we should be focusing on the processes that enable boundary making and remaking. ${ }^{15}$ This would entail considering the situations when boundaries might be considered more fluid (and why) and instances when these might seem impenetrable. It would also involve identifying how these perceptions vary depending on the group or the individual.

In this rapid report, we have attempted to explore these different perceptions of boundaries by analysing the experiences of joint research office staff and other actors in the context of a pandemic. We considered the instances when boundaries were considered more fluid by staff and the processes that led to these perceptions. We also identified situations when staff felt barriers remained rigid and even that new barriers had emerged. The PIs reflected mainly on positive experiences with the changes made in the joint research office, as the review and approval of studies appeared to be faster. However, some PIs also reflected on the large scale and breadth of the organisation's research portfolio prior to the pandemic that they perceived prevented them from gaining prompt access to joint research office staff and the timely review of research, as well as the perception of permanence of parts of the review process that ultimately reinforce or strengthen boundaries.

Our study showed that, in light of the unprecedented nature of the conditions created by the pandemic, the boundaries that had been previously established to set up distinct groups and responsibilities could be modified to allow for different ways of working. This led to the creation of new groups with different memberships, closer contact between staff from the joint research office and changes in interactions between staff from the joint research office, PIs and staff in the support departments. Some of the new structures and processes that were seen positively brought groups that had previously worked at a distance closer together. This led to the reconceptualisation of relations within the research system, adding more fluidity, close working interactions and an expanded team ethos.

Study participants reflected on the benefits of this reconceptualisation of relations and indicated that the closer 
working relationships established during the pandemic should remain. This could entail developing strategies to involve PIs more actively in the review process, establishing opportunities for the collaborative review of studies (creating group discussions comprising staff members from different departments of the joint research office as well as staff from support departments), and running more stages of review in parallel, rather than on a sequential basis (to save time).

The study is limited in the sense that it explored the experiences of a single NHS foundation trust and University research system over a short period of time and included a small number of telephone interviews. Future research will need to develop a longitudinal approach to the study of boundaries to explore how these change over time. This longitudinal approach would allow the identification of changes made under the conditions of the pandemic that could remain and be integrated into routine ways of working in a post-pandemic era. A comparison of the experiences across joint research offices in different settings would also allow the exploration of variability produced by the local context and the identification of factors that might be generalisable.

\section{Funding}

The study was supported by the National Institute for Health Research University College London Hospitals Biomedical Research Centre.

\section{References}

1 Middaugh MF. An empirical evaluation of boundary spanning as a conceptual framework for examining the organizational roles of offices of institutional research. Association for Institutional Research, 1984

2 McNally N. Research leadership - You've got to work at, and beyond, the boundaries. UCLH Biomedical Research Centre, 2020. www.uclhospitals.brc.nihr.ac.uk/latest-blogs/research-leadershipyou $\%$ E2 \% 80\% 99ve-got-work-and-beyond-boundaries.

3 Long JC, Cunningham FC, Braithwaite J. Bridges, brokers and boundary spanners in collaborative networks: a systematic review. BMC Health Serv Res 2013;13:158.
4 Hirst G, Mann L. A model of R\&D leadership and team communication: the relationship with project performance. $R \& D$ Management 2004:34:147-60.

5 Takanashi C, Lee K-J. Boundary spanning leadership, resource mobilisation, and performance of university-industry R\&D projects: a study in a Japanese university. Technol Anal Strateg Manag 2019;31:140-54.

6 Dey C, Ganesh MP. Team boundary activity: A review and directions for future research. Team Perform Manag 2017;23:273-92.

7 Johnson G, Vindrola-Padros C. Rapid qualitative research methods during complex health emergencies: A systematic review of the literature. Soc Sci Med 2017;189:63-75.

8 Vindrola-Padros C. Rapid ethnographies: A practical guide. University of Cambridge Press, 2020.

9 Green J, Thorogood N. Qualitative methods for health research. London: SAGE, 2013.

10 Harris KJ, Jerome NW, Fawcett SB. Rapid assessment procedures: A review and critique. Human Organization 1997;56:375-8.

11 Beebe J. Basic concepts and techniques of rapid appraisal. Human Organization 1995;54:42-51.

12 Gale NK, Heath G, Cameron E, Rashid S, Redwood S. Using the framework method for the analysis of qualitiative data in multidisciplinary health research. BMC Med Res Methodol 2013;13:117.

13 Vindrola-Padros C, Chisnall G, Cooper S et al. Carrying out rapid qualitative research during a pandemic: emerging lessons from COVID-19. Qual Health Res 2020;30:2192-204.

14 Barth F. Boundaries and connections. In Cohen A (ed), Signifying identities: Anthropological perspectives on boundaries and contested values. Routledge, 2012.

15 Cohen A (ed). Signifying identities: Anthropological perspectives on boundaries and contested values. Routledge, 2012.

Address for correspondence: Dr Cecilia Vindrola-Padros, Department of Targeted Intervention, University College London, Charles Bell House, 43-45 Foley Street, London W1W 7TY, UK.

Email: c.vindrola@ucl.ac.uk Twitter: @CeciliaVindrola 